\title{
A massive star origin for an unusual helium-rich super- nova in an elliptical galaxy
}

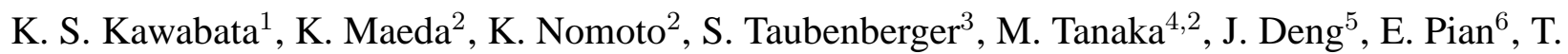
Hattori $^{7} \&$ K. Itagaki ${ }^{8}$

Accepted by Nature (24 March 2010)

\author{
${ }^{1}$ Hiroshima Astrophysical Science Center, Hiroshima University, 1-3-1 Kagamiyama, Higashi-Hiroshima, Hiroshima \\ 739-8526, Japan. \\ ${ }^{2}$ Institute for the Physics and Mathematics of the Universe (IPMU), University of Tokyo, 5-1-5 Kashiwanoha, \\ Kashiwa, Chiba 277-8583, Japan. \\ ${ }^{3}$ Max-Planck-Institut für Astrophysik, Karl-Schwarzschild-Straße 1, 85741 Garching, Germany. \\ ${ }^{4}$ Department of Astronomy, School of Science, University of Tokyo, Bunkyo-ku, Tokyo 113-0033, Japan. \\ ${ }^{5}$ National Astronomical Observatories, CAS, 20A Datun Road, Chaoyang District, Beijing 100012, China. \\ ${ }^{6}$ INAF Osservatorio Astronomico di Trieste, Via Tiepolo 11, I-3413 Trieste, Italy. \\ ${ }^{7}$ Subaru Telescope, National Astronomical Observatory of Japan, Hilo, HI 96720. \\ ${ }^{8}$ Itagaki Astronomical Observatory, Teppo-cho, Yamagata 990-2492, Japan.
}

The unusual helium-rich (type Ib) supernova SN 2005E is distinguished from any supernova hitherto observed by its faint and rapidly fading light curve, prominent calcium lines in latephase spectra and lack of any mark of recent star formation near the supernova location. These properties are claimed to be explained by a helium detonation in a thin surface layer of an accreting white dwarf (ref. 1). Here we report on observations of SN $2005 \mathrm{cz}$ appeared in an elliptical galaxy, whose observed properties resemble those of $\mathrm{SN} 2005 \mathrm{E}$ in that it is 
helium-rich and unusually faint, fades rapidly, shows much weaker oxygen emission lines than those of calcium in the well-evolved spectrum. We argue that these properties are best explained by a core-collapse supernova at the low-mass end $\left(8-12 M_{\odot}\right)$ of the range of massive stars that explode (ref. 2). Such a low mass progenitor had lost its hydrogen-rich envelope through binary interaction, having very thin oxygen-rich and silicon-rich layers above the collapsing core, thus ejecting a very small amount of radioactive ${ }^{56} \mathrm{Ni}$ and oxygen. Although the host galaxy NGC 4589 is an elliptical, some studies have revealed evidence of recent starformation activity (ref. 3), consistent with the core-collapse scenario.

We discovered SN $2005 \mathrm{cz}$ on 2005 July 17.5 UT in the elliptical galaxy NGC 4589. The spectrum of SN $2005 \mathrm{cz}$ taken on July 28 is well consistent with post-maximum spectra of type Ib supernovae $(\mathrm{SNe} \mathrm{Ib})^{4}$. Thus, $\mathrm{SN} 2005 \mathrm{cz}$ would originate from a core-collapse of an envelopestripped massive star. We tentatively assume that the epoch of our first spectrum is at $t=+26$ days, where $t$ is time after the maximum brightness (Fig. 1; see SI $§ 1$ ).

The late-time spectrum of SN $2005 \mathrm{cz}$ at $t=+179$ days is very unique; unlike most of other SNe Ibc/IIb SN 2005cz shows much stronger [Ca II] $\lambda \lambda 7291,7323$ than [O I] $\lambda \lambda 6300,6364$ (Fig. 2; see ref. 12, 13 for other $\mathrm{SNe}$ with large $\mathrm{Ca} / \mathrm{O}$ and $\mathrm{SI} \S 3$ for comparative discussion.). Oxygen is ejected mostly from the oxygen layer formed during the hydrostatic burning phase; its mass depends sensitively on the progenitor mass and is smaller for lower-mass progenitors. On the other hand, Ca is explosively synthesized during the explosion. Theoretical models predict that the stars having main-sequence masses of $M_{\mathrm{ms}}=13 M_{\odot}$ and $18 M_{\odot}$ produce 0.2 and $0.8 M_{\odot}$ of $\mathrm{O}$, and 
0.005 and $0.004 M_{\odot}$ of $\mathrm{Ca}$, respectively (e.g., ref. 14). Therefore, the $\mathrm{Ca} / \mathrm{O}$ ratio in the $\mathrm{SN}$ ejecta is sensitive to the progenitor mass ${ }^{15,16}$. To produce the extremely large $\mathrm{Ca} / \mathrm{O}$ ratio, the mass of the progenitor star of SN $2005 \mathrm{cz}$ should be smaller than of any other SNe Ib reported to date. For both SNe 1993J and 1994I that show weaker [Ca II] than [O I] (Fig. 2), the progenitor's masses are estimated to be $\sim 12-15 M_{\odot}$ (ref. 17,18), which are the smallest among well-studied samples with $[\mathrm{Ca}$ II $]<$ [O I] (e.g., ref. 16,19,20; see also Supplementary Fig. 3). Thus, the progenitor mass of SN $2005 \mathrm{cz}$ is likely $\leq 12-15 M_{\odot}$.

SN 2005cz is intrinsically fainter than the well-studied SN Ic 1994I by $\Delta R \sim 1.5$ mag (Fig. 3). In the pseudo bolometric light curve, the decline rate from the intermediate to the late phase is consistent with $\left(M_{\mathrm{ej}, \odot}^{2} / E_{51}\right) \leq 1$, and the luminosity requires that $M\left({ }^{56} \mathrm{Ni}\right) \leq 0.005-0.02 \mathrm{M}_{\odot}$ (Fig. 4). Additionally, $\left(M_{\mathrm{ej}, \odot} / E_{51}\right) \sim 1$ is suggested from the line velocity (Fig. 4 legend). We thus estimate $M_{\mathrm{ej}, \odot} \leq 1$ and $E_{51} \leq 1$, indicating a small progenitor mass ( $\leq 12 M_{\odot} ;$ ref. 27,2 ).

To explain the above peculiarities, we suggest a star with $M_{\mathrm{ms}}=10-12 M_{\odot}$ as the most likely origin of SN $2005 \mathrm{cz}$. If such a star had been single, its mass (and thus its mass loss rate) would have been too small to lose most of its H-rich envelope. Thus this star must have been in a close binary system. Then it became a He star of $\sim 2.5 M_{\odot}$ after undergoing Roche lobe overflow. This He star formed a $\mathrm{C}+\mathrm{O}$ core of $\sim 1.5 M_{\odot}$, which marginally exceeded the lower mass limit to form a Fe core ${ }^{28,29}$. The overlying He layer had $\sim 1 M_{\odot}$. Eventually, the He star underwent Fe core-collapse to explode as a SN Ib, leaving a $\sim 1.5 M_{\odot}$ neutron star behind. The ejecta had $\sim 1 M_{\odot}$, consistent with the observed constraint. The ejecta consists mostly of unburned 
material in the He layer and a small amount of explosively synthesized elements. The explosive burning products contain some $\mathrm{Fe}, \mathrm{Ca}, \mathrm{S}$, and $\mathrm{Si}$, but not much oxygen. Also the ejected part of the unburned oxygen-rich layer is extremely small. This scenario can explain the peculiar nebular spectrum with large $[\mathrm{Ca} \mathrm{II}] /[\mathrm{O} \mathrm{I}]$ ratio, as well as the low luminosity and its relatively rapid decrease.

An alternative candidate of the progenitor is a star with $M_{\mathrm{ms}} \sim 8-10 M_{\odot}$ in a close binary system. Such a star forms an electron-degenerate ONeMg core and undergo electron-captureinduced collapse ${ }^{29}$. The most likely scenario to realize a SN Ib would be the merging of an $\mathrm{ONeMg}$ white dwarf and a He white dwarf. The delay time between the star formation and the merging could be long enough to explain the origin of both $\mathrm{SN} 2005 \mathrm{cz}$ and recently reported $2005 \mathrm{E}^{1}$ with this scenario.

As for the host galaxy problem, the $\sim 10 M_{\odot}$ star model is found to be consistent with the properties recently-inferred for the host galaxy of SN 2005cz. It is still a genuine E2 galaxy ${ }^{30}$, but has a relatively young stellar population with life times of $\sim 10^{7}-10^{8}$ years $^{3}$ and $\mathrm{SN}$ Ib $2005 \mathrm{cz}$ is likely the end product of one of these young stars (See SI §2).

The mass range of 10-12 $M_{\odot}$ has not been theoretically investigated in much detail so far, but, as SN 2005cz suggests, the SNe resulting from these stars may have a very special abundance pattern in the ejecta and play an important role in the chemical evolution of galaxies (see SI $\S 3$ ). 
1. Perets, H. B. et al., A new type of stellar explosion, Nature, submitted, arXiv:0906.2003 (2009).

2. Smartt, S. J., Progenitors of core-collapse supernovae, Ann. Rev. Astron. Astrophys., 47, 63106 (2009).

3. Zhang, Y., Gu, Q.-S. \& Ho, L. C., Stellar and dust properties of local elliptical galaxies: clues to the onset of nuclear activity, Astron. Astrophys. 487, 177-183 (2008).

4. Leonard, D. C., Supernova 2005cz in NGC 4589, IAU Circ. 8579 (2005).

5. Branch, D. et al., Direct Analysis of Spectra of Type Ib Supernovae, Astrophys. J. 566, 10051017 (2002).

6. Barbon, R. et al., SN 1993J in M81: One year of observations at Asiago, Âstron. Astrophys. Suppl. 110, 513-519 (1995).

7. Filippenko, A. V. et al., The Type Ic supernova 1994I in M51: Detection of helium and spectral evolution, Astrophys. J. 450, L11-L15 (1995).

8. Sauer, D. N. et al., The properties of the 'standard' Type Ic supernova 1994I from spectral models, Mon. Not. R. Astron. Soc. 369, 1939-1948 (2006).

9. Maeda, K. et al., Asphericity in Supernova Explosions from Late-Time Spectroscopy, Science 319, 1220-1223 (2008).

10. Phillips, M. M. et al., The peculiar SN 2005hk: Do some type Ia supernovae explode as deflagrations?, Publ. Astron. Soc. Pacif. 119, 360-387 (2007). 
11. Valenti, S. et al., A low energy core-collapse supernova without a hydrogen envelope, Nature 459, 674-677 (2009).

12. Filippenko, A. V. et al., Supernovae 2001co, 2003H, 2003dg, and 2003dr, IAU Circ. 8159, 2 (2003).

13. Foley, R. J. et al., SN 2008ha: An extremely low luminosity and exceptionally low energy supernova, Astron. J. 138, 376-391 (2009).

14. Nomoto, K., Tominaga, N., Umeda, H., Kobayashi, C. \& Maeda, K., Nucleosynthesis yields of core-collapse and hypernovae, and galactic chemical evolution, Nucl. Phys. A, 777, 424-458 (2006).

15. Fransson, C. \& Chevalier, R. A., Late emission from supernovae: A window on stellar nucleosynthesis, Astrophys. J. 343, 323-342 (1989).

16. Maeda, K. et al., SN 2006aj Associated with XRF 060218 at Late Phases: Nucleosynthesis signature of a neutron-driven explosion, Astrophys. J. 658, L5-L8 (2007).

17. Shigeyama, T. et al., Theoretical light curves of type IIb Supernova 1993J, Astrophys. J. 420, 341-347 (1994).

18. Nomoto, K. et al., A carbon-oxygen star as progenitor of the type-Ic supernova 1994I, Nature 371, 227-229 (1994).

19. Mazzali, P. A., Nomoto, K., Patat, F. \& Maeda, K., The nebular spectra of the hypernova SN 1998bw and evidence for asymmetry, Astrophys. J. 559, 1047-1053 (2001). 
20. Mazzali, P. A. et al., The aspherical properties of the energetic type Ic SN 2002ap as inferred from its nebular spectra, Astrophys. J. 670, 592-599 (2007).

21. Richmond, M. W. et al., $U B V R I$ photometry of the type Ic SN 1994 I in M51, Astrophys. J. 111, 327-339 (1996).

22. Stritzinger, M. et al., The He-rich Core-collapse Supernova 2007Y: Observation from X-ray to Radio Wavelengths, Astrophys. J. 696, 713-728 (2009).

23. Botticella, M. T. et al., SN 2008S: an electron capture SN from a super-AGB progenitor?, Mon. Not. R. Astron. Soc., accepted, arXiv: 0903.1286 (2009).

24. Iwamoto, K. et al., Theoretical light curves for the Type Ic Supernova SN 1994I, Astrophys. J. 437, L115-L118 (1994).

25. Patat, F. et al., The Metamorphosis of SN 1998bw, Astrophys. J. 555, 900-917 (2001).

26. Yoshii, Y. et al., The optical/near-infrared light curves of SN 2002ap for the first 140 days after discovery, Astrophys. J. 592, 467-474 (2003).

27. Tanaka, M. et al., Nebular phase observations of the Type Ib Supernova 2008D/X-ray transient 080109: Side-viewed bipolar explosion, Astrophys J. 700, 1680-1685 (2009).

28. Nomoto, K. \& Hashimoto, M., Presupernova evolution of massive stars, Phys. Rep. 163, 13-36 (1988).

29. Nomoto, K., Evolution of 8-10 solar mass stars toward electron capture supernovae. I - Formation of electron-degenerate O + Ne + Mg cores, Astrophys. J. 277, 791-805 (1984). 
30. Hakobyan, A. A. et al. 2008, Early-type galaxies with core collapse supernovae, Astron. Astrophys. 488, 523-531 (2008).

Acknowledgements We would like to thank D. Leonard for permission to publish his early-time spectrum of SN 2005cz. We also thank S. Valenti for permission to use their spectra of SN 2008ha. We gratefully acknowledge advice and help from P. A. Mazzali through a series of this study. This work is based on observations collected at the 2.2-m Telescope at the Calar Alto Observatory (Sierra de Los Filabres, Spain), at the Keck Telescope, and at Subaru Telescope (operated by the National Astronomical Observatory of Japan). We are grateful to the staff members at the observatories for their excellent assistance, especially to T. Sasaki, K. Aoki, G. Kosugi, T. Takata and M. Iye. This research is supported by World Premier International Research Center Initiative (WPI Initiative), MEXT, Japan, and by the Grant-in-Aid for Scientific Research of the JSPS and MEXT. M.T. is supported through the JSPS (Japan Society for the Promotion of Science) Research Fellowship for Young Scientists. J.D. is supported by the NSFC and by the 973 Program of China.

Author Contributions K.S.K., K.M., K.N., J.D. and E.P. organized the observations and discussion. K.M., K.N and K.S.K. have written the manuscript. K.S.K., S.T., and K.I. are responsible for data acquisition and reduction; J.D. and E.P. were the PIs of the relevant Subaru programs, S05B-132 and S05B-054, respectively. M.T., S.T. contributed to discussions. T.H. provided expertise on data acquisition at Subaru Telescope.

Competing Interests The authors declare that they have no competing financial interests.

Correspondence Correspondence and requests for materials should be addressed to K.S.K. (email: 
kawabtkj@hiroshima-u.ac.jp).

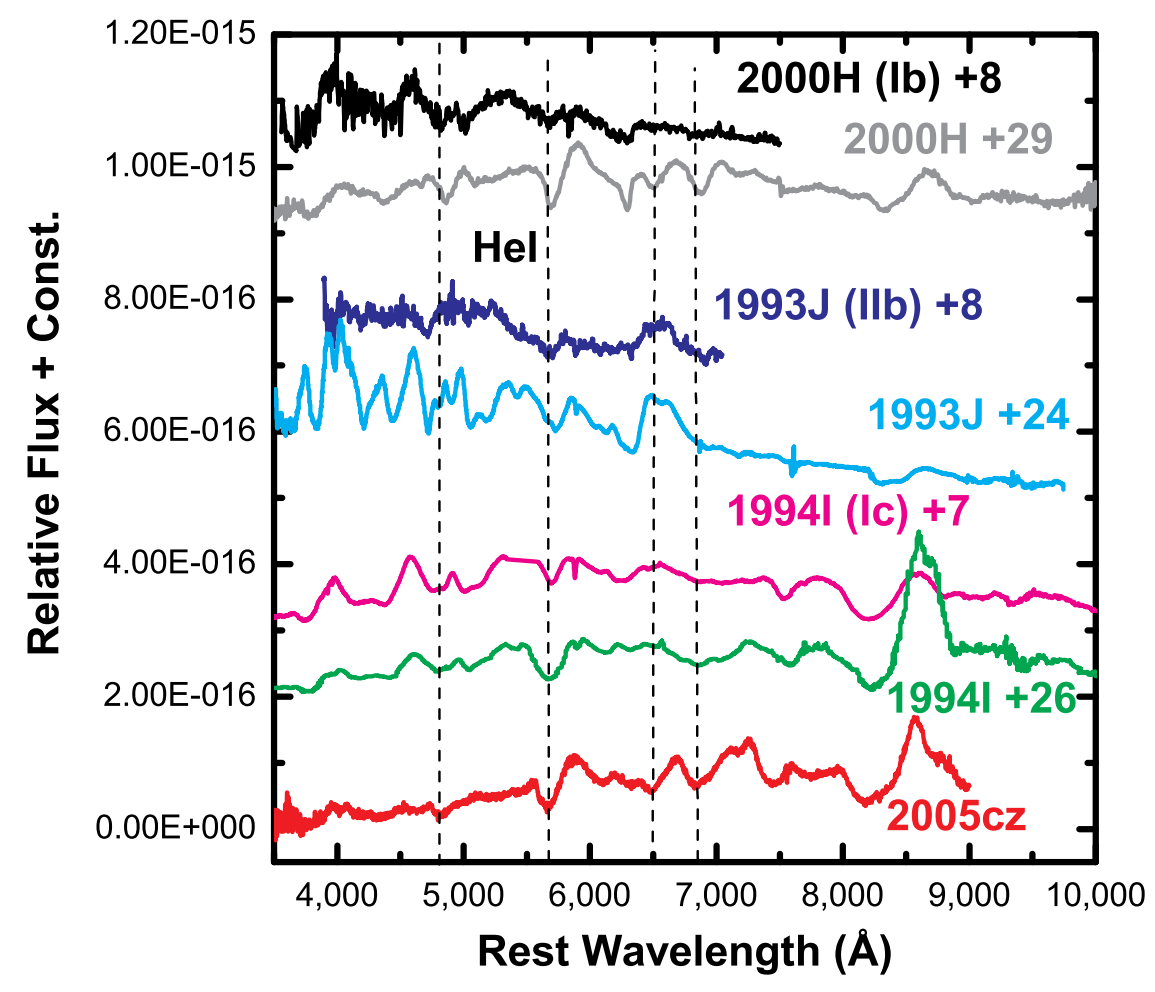




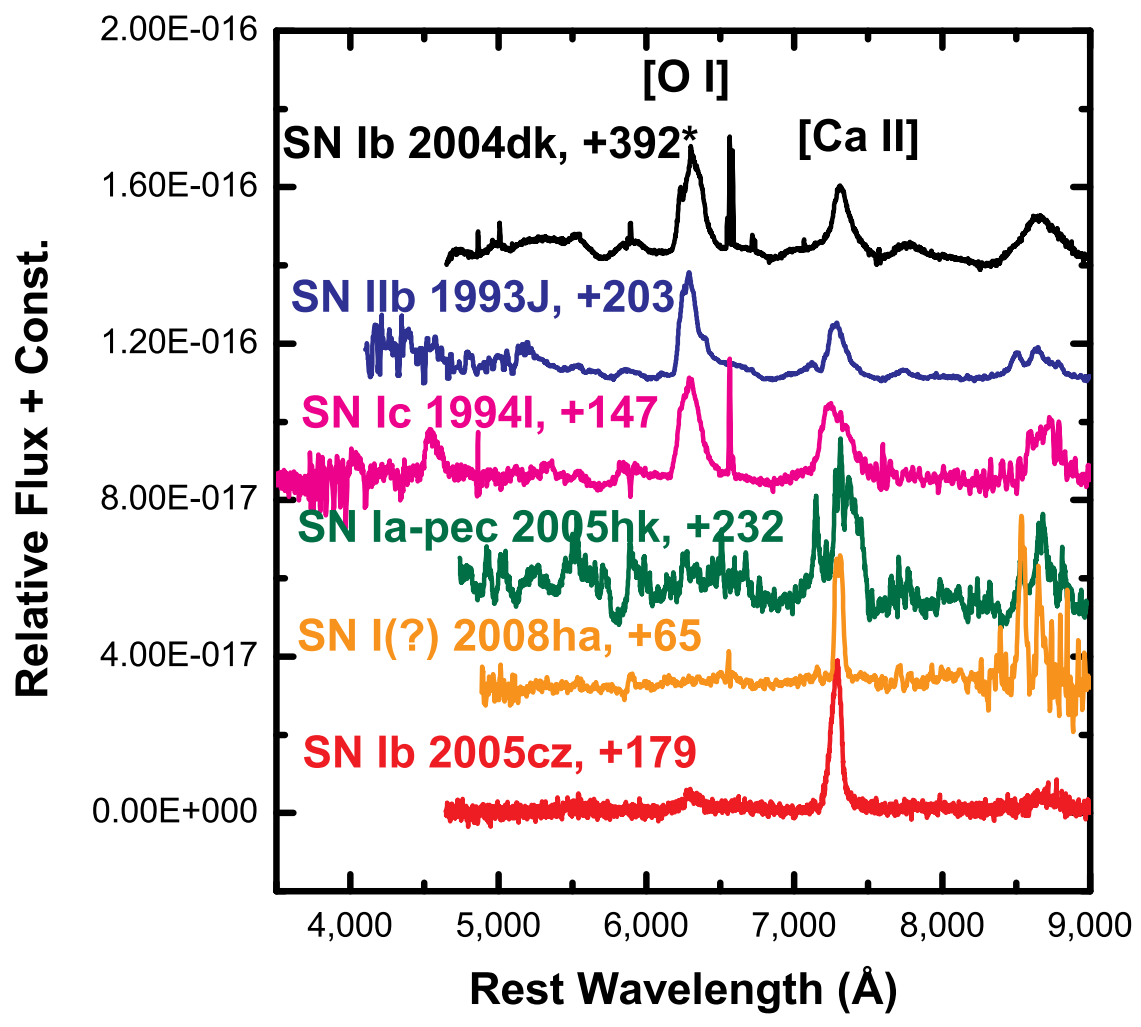




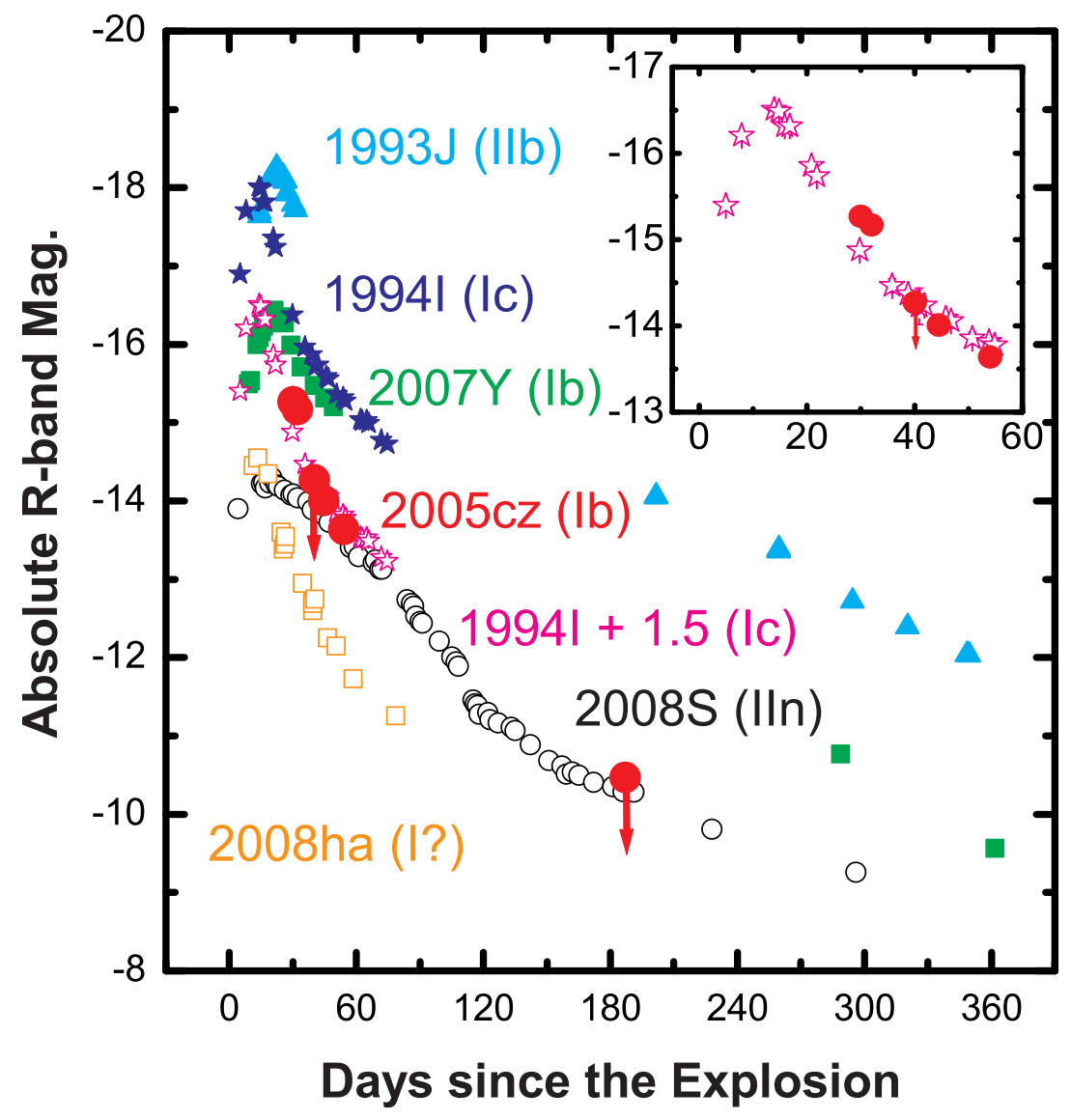




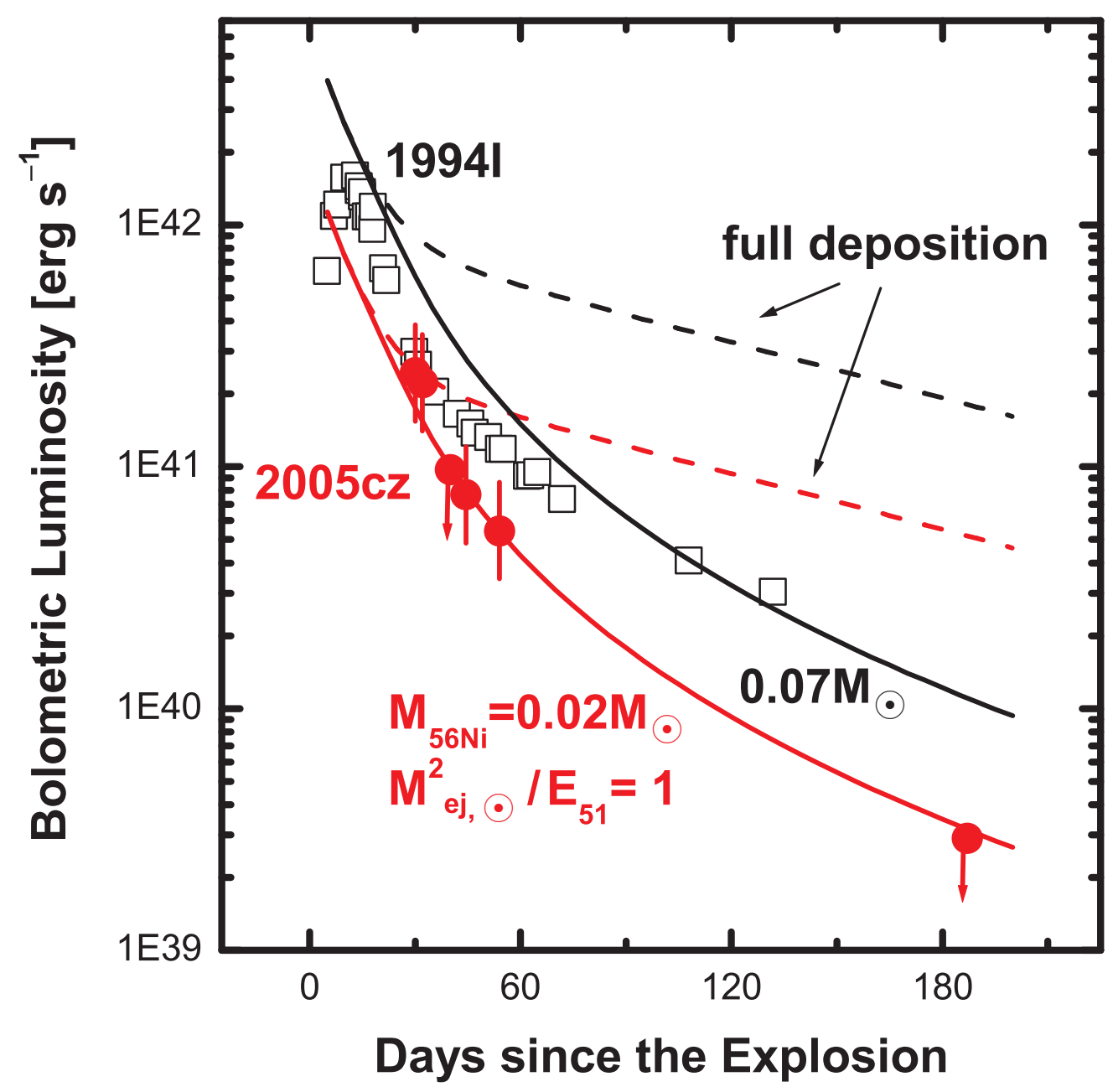


Figure 1 Early-time spectrum of SN Ib 2005cz compared with other envelope-stripped SNe at similar phases. The spectrum of SN $2005 \mathrm{cz}$ (red) is taken on $2005 \mathrm{Jul} 28$. Also shown are the spectra of $\mathrm{SN} \mathrm{lb} 2000 \mathrm{H}$ at $t=+8$ (black) and $t=+29$ days (gray) ${ }^{5}$, SN Illb 1993J at $t=+8$ days (blue) and $t=+24$ days (cyan) ${ }^{6}$, and SN Ic 1994I at $t=+7$ days (magenta) and $t=+26$ days (green) $)^{7,8}$. The $\mathrm{SN} \mathrm{lb}$ is characterized by strong helium lines and weak silicon lines, while in the SN Ic both helium and silicon lines are weak. The SN Ilb shows a SN II-like spectrum characterized by the strong hydrogen features at early times, and becomes SN Ib/c-like at late times. All these SNe are thought to have partly or fully stripped off their outer layers of hydrogen and helium before the explosions. The overall appearance of spectral features in SN $2005 \mathrm{cz}$ is quite similar to those of the $\mathrm{SN} \mathrm{Ib} 2000 \mathrm{H}$ at $t=+29$ days, the SN IIb 1993J at $t=+24$ days (despite its stronger $\mathrm{H}$ lines), and also the typical SN Ic 1994I at $t=+26$ days (despite its lack of the strong He lines). The spectra are corrected for the host redshift and the reddening. We adopted a total (Milky Way + host) reddening of $E(B-V)=0.13(0.03+0.1)$ mag in SN 2005cz, 0.23 $(0.23+0.0) \mathrm{mag}$ in $\mathrm{SN} 2000 \mathrm{H}, 0.45 \mathrm{mag}$ in SN 1994I, and $0.3 \mathrm{mag}$ in SN 1993J. The flux is on an absolute scale for SN $2005 \mathrm{cz}$, calibrated with the Calar Alto photometry obtained four nights later. For the comparison $\mathrm{SNe}$, the fluxes are on an arbitrary scale and constants are added for presentation. The positions of the prominent $\mathrm{He}$ I lines are shown by the dotted lines. The spectrum of SN $2005 \mathrm{cz}$ is well consistent with the post-maximum spectra of SNe lb.

Figure 2 Calcium-rich late-time spectrum of SN lb 2005cz. It is taken on 2006 Dec 
$27\left(t=+179\right.$ days). Also shown are SN Ib 2004dk at $t \sim 390$ days $^{9}, \mathrm{SN}$ Ilb 1993J at $t=+203$ days $^{6}$, SN Ic 1994I at $t=+147$ days $^{8}$, peculiar SN la 2005hk at $t=+232$ days $^{10}$, and peculiar SN I(?) 2008ha at $t=+65$ days ${ }^{11}$. As time goes by, the ejecta become transparent to optical light, following the expansion and density decrease. Late-time spectra of $\mathrm{SNe} \mathrm{lb} / \mathrm{c}$ are thus characterized by various emission lines, mostly of forbidden transitions. The spectrum of SN 2004dk is typical for SNe lb/c at late times (e.g., see fig.2 of ref. 9). The spectra are corrected for the host redshift, but not for reddening. The flux is on an approximate absolute scale for SN $2005 \mathrm{cz}$, calibrated with the spectroscopic standard star (but not with photometry), while it is on an arbitrary scale for the comparison SNe. The asterisk of SN 2004dk denotes the days since its discovery (not maximum light). It is very unique that SN 2005cz shows only weak [O I] $\lambda \lambda 6300,6364$ and much stronger [Ca II] $\lambda \lambda 7291,7323$ than [O I]. The relatively weak Ca II IR triplet compared with other $\mathrm{SNe}$ might suggest a lower density ejecta of SN 2005cz. It is interesting that the [Ca II] line is considerably narrow (half-width at half-maximum $0.005 c$ ) compared with the blueshift of the absorption in Ca II IR triplet in the early-time spectrum $(\sim 0.04 c)$.

Figure 3 Absolute $R$-band light curve of rapidly-fading SN lb $2005 \mathrm{cz}$. It is shown by filled red circles and compared with those of SN Ilb 1993J (cyan triangles), SN Ic 1994I (blue stars), SN Ib 2007Y (green squares), SN IIn 2008S (black open circles), and SN I (?) 2008ha (orange open squares). Also shown is the light curve of SN 1994I, but dimmed by 1.5 magnitudes (magenta open stars). For SN $2005 \mathrm{cz}$, the first three points denote unfiltered magnitudes which are approximately $R$-band magnitudes. The two points with 
downward arrows are $3 \sigma$ upper-limits. The distance moduli and total reddening values are taken as follows: $[\mu, \mathrm{E}(\mathrm{B}-\mathrm{V})]=(32.23 \mathrm{mag}, 0.13 \mathrm{mag})$ for $2005 \mathrm{cz}$ (see SI $\S 1),(27.8$ mag, $0.3 \mathrm{mag}$ ) for 1993J, (29.6 mag, $0.45 \mathrm{mag}$ ) for 1994I, (31.43 mag, $0.112 \mathrm{mag}$ ) for 2007Y, (31.55 mag, $0.076 \mathrm{mag}$ ) for $2008 \mathrm{ha}$, and (28.78 mag, $0.687 \mathrm{mag})$ for $2008 \mathrm{~S}$. We assume $R_{V}=3.1$ to convert the colour excess to the $R$-band extinction. The data points, as well as the distance and the reddening, are from the literature ${ }^{6,11,21,22,23}$. The putative explosion date for $\mathrm{SN} 2005 \mathrm{cz}$ is assumed to be 2005 Jun 17,30 days before the discovery and 15 days before maximum brightness (SI $\S 1$ ). The LC tail of SN $2005 \mathrm{cz}$ is similar to those of SN IIn 2008S and SN Ic 1994I (dimmed by $1.5 \mathrm{mag}$ ). From this, we estimate the mass of ${ }^{56} \mathrm{Ni}$ as $M\left({ }^{56} \mathrm{Ni}\right)=10^{-1.5 / 2.5} \times 0.07 \mathrm{M}_{\odot} \sim 0.018 \mathrm{M}_{\odot}\left(M\left({ }^{56} \mathrm{Ni}\right)=0.07 \mathrm{M}_{\odot}\right.$ is for $\mathrm{SN}$ $\left.\left.1994\right|^{24}\right)$.

Figure 4 Pseudo bolometric light curve of SN Ib 2005cz suggests that the ejecta has a low mass, low kinetic energy, and a tiny amount of ${ }^{56} \mathrm{Ni}$. The light curve (filled red circles) is compared with a simple $\gamma$-ray and positron deposition model with $M\left({ }^{56} \mathrm{Ni}\right)=0.02 \mathrm{M}_{\odot}$ and $M_{\mathrm{ej}, \odot}^{2} / E_{51}=1$ (red line), where $E_{51}$ is the kinetic energy $E_{\mathrm{K}}$ measured in unit of $10^{51}$ ergs. We also plot the bolometric light curve of SN Ic 1994I (open black squares) ${ }^{21}$ and a simple deposition model with $M\left({ }^{56} \mathrm{Ni}\right)=0.07 \mathrm{M}_{\odot}$ (black line) for comparison. Except for the last point (upper-limit), we simply assume the bolometric correction $B C \equiv$ $M_{\mathrm{Bol}}-M_{R}=0.5$, derived from SNe 1998bw, 2002ap and 2008D at similar epochs ${ }^{25,26,27}$. As this is a very crude estimate, we adopt an error bar of \pm 0.5 mag for the bolometric luminosity. The deposition models adopt the $\gamma$-ray opacity for the Compton scattering 
$\left(\tau_{\gamma} \propto M_{\mathrm{ej}}^{2} E_{\mathrm{K}}^{-1} t^{-2}\right)$ and assuming the full deposition of positrons. The decline rate from the intermediate to the late phase is consistent with $\left(M_{\mathrm{ej}, \odot}^{2} / E_{51}\right) \leq 1$. Combining this expression with $\left(M_{\mathrm{ej}, \odot} / E_{51}\right) \sim 1$ as indicated by the similarity in the absorption velocity seen in SN 2005cz and those in SNe 1993J and 1994I (Fig. 1, Supplementary Fig. 1), we estimate $M_{\mathrm{ej}, \odot} \leq 1$ and $E_{51} \leq 1$. The luminosity requires that $M\left({ }^{56} \mathrm{Ni}\right) \leq 0.02 \mathrm{M}_{\odot}$. Note that the estimate for $M\left({ }^{56} \mathrm{Ni}\right)$ is sensitively affected by the explosion date. The upper limit to $M\left({ }^{56} \mathrm{Ni}\right)$ is only $M\left({ }^{56} \mathrm{Ni}\right) \leq 0.005 \mathrm{M}_{\odot}$, if the explosion date is as late as $2005 \mathrm{Jul} 15$ (just a few days before the discovery). 


\section{Supplementary Information}

\section{Observation}

We discovered SN $2005 \mathrm{cz}$ on 2005 July 17.5 UT at $13^{\prime \prime}$ offset from the nucleus of the elliptical galaxy NGC 4589. No object brighter than 18.5 mag was visible at the SN position on 2005 June 20 (ref. 31).

The discovery and subsequent unfiltered images were taken with a 0.6-m reflector and a CCD (Kodak KAF-1001E) at Itagaki Astronomical Observatory (IAO) in Yamagata, Japan. The derived magnitudes are approximately consistent with $R$ magnitudes.

Other imaging observations were performed by the Calar Alto 2.2-m telescope (CA2.2) equipped with the Calar Alto Faint Object Spectrograph (CAFOS) in $B, V, R$ and $I$ bands, and by the 8.2-m Subaru Telescope equipped with the Faint Object Camera and Spectrograph (FOCAS ${ }^{32}$ ) in $B$ and $R$ bands. Imaging observations with Subaru were done in photometric conditions; standard stars around PG 1525-071 in August 2005 and around PG 0942-029 in December 2005 were observed for photometric calibration.

The data reduction was performed using the IRAF package DAOPHOT. Since SN $2005 \mathrm{cz}$ was close to the bright core of the host galaxy, we subtracted the host galaxy component prior to the photometry for more reliable photometry. For the unfiltered images at IAO, we used the pilot survey image taken with the same system on 2005 May 25 as 
the host template 1 For the CA2.2 and Subaru images, we took data of the same field, as well as the standard stars around PG 0918+029, by CA2.2+CAFOS in a photometric night on 2009 Feb 19 and used them as the host galaxy template after point-spread-functionmatching.

The derived $R$ (and unfiltered) magnitudes are shown in the Supplementary Table 1. The other magnitudes are $B=21.18 \pm 0.30 \mathrm{mag}, V=19.69 \pm 0.18 \mathrm{mag}, I=17.72 \pm 0.11$ mag on 2005 Aug 1, and $B=21.0 \pm 0.2$ mag on 2005 Aug 10. On 2005 Jul 27 and Dec 27, the SN was not detected and we just derived $3 \sigma$ upper-limit for the SN luminosity. For the Dec 27 data, we derived more reliable upper-limit of the bolometric luminosity (Fig. 4) as follows; first, we subtracted a continuum from the Subaru spectrum and then scaled it to the observed upper-limit $(R>22.1)$. We then integrated the flux at optical wavelengths. Finally, we assumed that the NIR contribution was $30 \%$ of the optical luminosity, a typical value seen in $\mathrm{SNe} \mathrm{lb} / \mathrm{c}$ at late phases ${ }^{33}$.

The early-time spectrum of SN $2005 \mathrm{cz}$ was obtained on 2005 Jul 28 UT with the 10m Keck I Telescope equipped with the Low-Resolution Imaging Spectrometer (LRIS ${ }^{34}$ ). The total exposure time was $500 \mathrm{~s}$. The seeing was $\sim 1^{\prime \prime} .2$ and the airmass was relatively large, $\sim 2.3$. The wavelength resolution measured from sky lines was $9 \AA$. The late-phase spectrum was obtained with Subaru+FOCAS on 2005 Dec 27 UT. The total exposure time was $1800 \mathrm{~s}$. The seeing was $\sim 1^{\prime \prime} .0$ and the airmass was $\sim 1.8$. The wavelength

\footnotetext{
${ }^{1}$ The discovery magnitude of 16.0 reported in IAUC 8569 included a large bias caused by the steep brightness distribution of the host galaxy core.
} 
resolution was $11 \AA$. These data were reduced with IRAF in a standard manner for longslit spectroscopy.

For the extinction within our Galaxy and the host galaxy, we adopted $E(B-V)=0.03$ and 0.1 mag, respectively. The former is inferred from the infrared dust map ${ }^{35}$, while the latter is estimated from the equivalent width of $\mathrm{Na}$ I $\mathrm{D}$ absorption feature in the earlyphase spectrum $(E W \leq 0.34 \AA)$ and an empirical formula ${ }^{36}$. Although the formula allows for a range of $E(B-V) \leq 0.044-0.13$ mag, the extinction within the inner $3^{\prime \prime}$ of NGC 4589 has been estimated to be $E(B-V) \sim 0.1$ mag by the spectrum template fitting ${ }^{37}$. Since the SN position is reasonably separated from the dusty bar near the nucleus of the host galaxy, the extinction should not be large there. Therefore, we take $E(B-V)=0.1$ mag as a reference value for the host extinction.

We assume that $t=+26$ days as the epoch of the first spectrum (Jul 28) because of the overall resemblance of the spectral features with SN Ic 1994I at $t=+26$ days and with SN Ib $2000 \mathrm{H}$ at $t=+29$ days. This estimate still includes a large uncertainty; e.g., the He I line velocities of $\sim 9,000-12,000 \mathrm{~km} \mathrm{~s}^{-1}$ on Jul 28 (Supplementary Fig. 1) would be more typical for $\mathrm{SNe} \mathrm{lb}$ at $t=0$ to +10 days. Since the estimation for ${ }^{56} \mathrm{Ni}$ mass is sensitively affected by the explosion date, we also consider an extreme case in which the discovery was close to the explosion date, and give the possible range of $M\left({ }^{56} \mathrm{Ni}\right.$ ) (see Fig. 4 legend). Anyway, the choice here does not affect our main conclusions. 
Supplementary Table 1: Summary of observation of SN 2005cz

\begin{tabular}{llllll}
\hline \hline Date & MJD & Epoch & Telescope+ & Setup & $R$ \\
(UT) & & (days) & Instrument & & $(\mathrm{mag})$ \\
\hline 2005 May 25.7 & 53516.7 & - & IAO 0.6 & Imaging(unfiltered) & - \\
2005 Jul 17.5 & 53568.5 & +15.5 & IAO 0.6 & Imaging(unfiltered) & $17.3 \pm 0.1$ \\
2005 Jul 19.5 & 53570.5 & +17.5 & IAO 0.6 & Imaging(unfiltered) & $17.4 \pm 0.1$ \\
2005 Jul 27.5 & 53578.5 & +25.5 & IAO 0.6 & Imaging(unfiltered) & $>18.3$ \\
2005 Jul 28 & 53579 & +26 & KeckI+LRIS & Spectropolarimetry & - \\
2005 Aug 1.0 & 53583.0 & +30.0 & CA2.2+CAFOS & Imaging(BVRI) & $18.56 \pm 0.12$ \\
2005 Aug 10.3 & 53592.3 & +39.3 & Subaru+FOCAS & Imaging(BR) & $18.93 \pm 0.05$ \\
2005 Dec 27.6 & 53731.6 & +178.6 & Subaru+FOCAS & Imaging $(B R) /$ Spectroscopy & $>22.1$ \\
2009 Feb 19.0 & 54881.0 & - & CA2.2+CAFOS & Imaging(BVRI) & - \\
\hline
\end{tabular}

\section{Stellar population in the elliptical galaxy NGC 4589}

It is apparently puzzling that SN Ib $2005 \mathrm{cz}$ appears in the elliptical galaxy NGC 4589 if it is the Fe core-collapse event, because elliptical galaxies generally contain only low-mass, old population stars. Recently, Hakobyan et al. ${ }^{29}$ reexamined the morphology of the host galaxies of 22 core-collapse $\mathrm{SNe}$ (i.e., type II/lbc) which had been previously classified as Elliptical or S0 galaxies. They concluded that 19 cases were simply misclassifications of the host galaxy type. NGC 4589 remains a genuine E2 galaxy.

However, from the literature search related to NGC 4589, Hakobyan et al. pointed out that there is a Low lonization Nuclear Emission-line Region (LINER) activity (Type "L2"38), and suggested the host to be a merger remnant. There is also an evidence for 
unusual distribution of interstellar dust from HST and AKARI studies ${ }^{39,40}$, being consistent with the merger scenario. Thus, the appearance of the SN Ib in this particular early-type galaxy may not conflict with the general scenario of stellar evolution and explosion.

According to a population synthesis model for the integrated light from the host galaxy ${ }^{30}$, it has been suggested that about $90 \%$ of the host flux is contributed by an old population with life times longer than $10^{10}$ years (i.e., $M_{\mathrm{ms}} \leq 1 M_{\odot}$ ), whereas a relatively young population with life times $\sim 10^{7}-10^{8}$ years (i.e., $M_{\mathrm{ms}} \leq 10 M_{\odot}$ ) contributes to the remaining $\sim 10 \%$. Thus, it is likely that $\mathrm{SN} \mathrm{Ib} 2005 \mathrm{cz}$ is the end product of one of these young stars, which were produced by the galaxy merger about $\sim 10^{8}$ years ago.

\section{Progenitors of faint, Ca-rich supernovae}

In addition to $\mathrm{SN} 2005 \mathrm{cz}$, there are examples of a faint, hydrogen deficient, possibly corecollapse SN which shows the large Ca/O line ratios, e.g., SNe 1997D, 2005E, 2005cs, 2005hk, and 2008ha ${ }^{11,12,10,1}$. As discussed below, however, they have very different observational features except for the late-time $\mathrm{Ca} / \mathrm{O}$ ratio. Thus it may not be the case that the origin of the large $\mathrm{Ca} / \mathrm{O}$ ratio and the progenitors are the same for all these $\mathrm{SNe}$.

SN 2005hk belongs to a subclass of peculiar SNe la, SN 2002cx-like class ${ }^{9,41}$, characterized by low luminosities and low expansion velocities $\left(\sim 5,000 \mathrm{~km} \mathrm{~s}^{-1}\right)$. The earlyphase spectra of SN 2005hk are dominated by low-velocity permitted lines of Fe, without any resemblance to $\mathrm{SN} 2005 \mathrm{cz}$ whose velocity and spectral features are those of typical 
SNe Ib (Supplementary Fig. 2). Thus, it is unlikely that the progenitor of SN 2005hk is the same as SN $2005 \mathrm{cz}$.

The early spectra of SN 2008ha are similar to SN 2005hk, but show even much lower velocities $\left(\sim 2,000 \mathrm{~km} \mathrm{~s}^{-1} \text { for most lines, and } \sim 5,000 \mathrm{~km} \mathrm{~s}^{-1} \text { for Ca II IR }\right)^{10,12}$. No clear detection of $\mathrm{He}$ is reported. This is totally different from the early spectrum of SN $2005 \mathrm{cz}$ that shows strong He lines and the expansion velocity of $\sim 10,000 \mathrm{~km} \mathrm{~s}^{-1}$ (Supplementary Fig. 1). The low expansion velocity of SN 2008ha is consistent with the fall-back SN model with a massive, black-hole-forming progenitor rather than the less massive progenitor model ${ }^{44}$. It has also been claimed to be an explosion of a white dwarf like other SNe la based on detection of silicon and sulfur features in the early-phase ${ }^{45}$. Thus, the origin of SN 2008ha is still controversial.

Adding to this, we should note that the reported "late-time" spectra of SNe 2005hk and 2008ha are not fully nebular (Fig. 2), which is in contrast to the case of SN 2005cz. Thus, it is possible that the $\mathrm{O} / \mathrm{Ca}$ ratio is affected by the attenuation within the ejecta. This would imply that the weak (or absence of) [O I] in these SNe would not necessarily indicate the small O-layer. This effect may also appear in SN 2005E to some extent, given its relatively young age ( $\sim 2$ months) of the reported late-time spectrum ${ }^{1}$. Our late-time spectrum of $S N 2005 \mathrm{cz}$ seems genuinely nebular, and thus the large $\mathrm{Ca} / \mathrm{O}$ ratio is more clearly the case than in other examples (except for SNe II; see ref. 2 and below).

For SNe 2005hk and 2008ha, it is also not clear whether the strong Ca lines in 
the late-phases are emitted from the newly-synthesized materials. The velocities of the "nebular" lines are similar to those in the early-phase spectra ${ }^{12}$. In contrast, the velocity of the nebular Ca lines in SN 2005cz $\left(\sim 1,500 \mathrm{~km} \mathrm{~s}^{-1}\right)$ is much lower than that in the early-phase (Supplementary Fig. 1), suggesting that the Ca lines are emitted from the innermost region of the ejecta where the newly-synthesized Ca dominates the emission.

SNe 1997D and 2005cs are both faint SNe II with the slow expansion velocities ${ }^{46,47}$. Although they do show the large $\mathrm{Ca} / \mathrm{O}$ ratio in the nebular spectra, the comparison with SN Ib $2005 \mathrm{cz}$ should be done carefully. SNe II generally show the $\mathrm{Ca} / \mathrm{O}$ line ratio being larger than $\mathrm{SNe} \mathrm{lb} / \mathrm{c}$, since $\mathrm{Ca}$ in the $\mathrm{H}$-rich envelope can also contribute to $[\mathrm{Ca}$ II] and Ca II IR triplet (e.g., ref. 14). A low mass progenitor is favoured for SN 2005cs, while the progenitor of SN 1997D is still controversial. These progenitors may or may not be consistent with the $\mathrm{Ca} / \mathrm{O}$ line ratio in the nebular phase. Further study including the emission from the $\mathrm{H}$-rich envelope is necessary to use the $\mathrm{Ca} / \mathrm{O}$ line ratio as an indicator of the progenitor mass for SNe II.

Detailed composition structure should be the key to the understanding of the progenitor and explosion mechanism of SN 2005cz. Deriving the detailed abundance from the nebular spectrum, however, is highly model dependent (e.g., see the above discussion for SNe II). Unfortunately, there is no strong Si or S line in optical wavelengths in the nebular phase, which could in principal be used to discriminate different scenarios.

The existence of $2005 \mathrm{cz}$-like $\mathrm{SNe}$, which have ejected material with little $\mathrm{O}$ and a 
relatively large amount of $\mathrm{Ca}$, may have important implications to the chemical evolution of galaxies. In our Galaxy, a very Ca-rich, extremely metal-poor (EMP) halo star has recently been discovered ${ }^{48}$. Such an EMP star may have formed from the debris of $2005 \mathrm{cz}$-like SNe. It would also be interesting to note that some dwarf galaxies contain EMP stars whose abundance ratios between the alpha-elements and Fe are much smaller than the halo stars ${ }^{49,50}$. The oxygen-poor $2005 \mathrm{cz}$-like SNe might be related to the formation of such EMP stars.

Current theoretical models still have lots of uncertainties and further observational constraints are necessary to fully understand the final stage of the evolution of stripped stars of different masses (See also Supplementary Fig. 3). The evolutionary scenario of 10-12 $M_{\odot}$ we propose in this paper (paragraph 5-6) is indeed similar to those have applied for ordinary SNe Ibc from more massive than $12 M_{\odot}$. However, it is a new theoretical argument that $10-12 M_{\odot}$ low mass models can have distinct properties of low $M\left({ }^{56} \mathrm{Ni}\right)$ production, low explosion energy, and the large $\mathrm{Ca} / \mathrm{O}$ (see ref. 2 for the similar conclusions from observations of SNe II). These are quite different from more massive models, and consistent with the new observation of SN 2005cz. Also, our suggestion to connect the explosions in the ONeMg white dwarfs with $\mathrm{SNe} \mathrm{lb}$ is quite new (see also ref. 1). We also note that our discovery of $\mathrm{SN} 2005 \mathrm{cz}$ and the faint nature of the 10-12 $M_{\odot}$ binary SN may solve the puzzle why SNe lb from 10-12 $M_{\odot}$ binary stars have not been observed. Even though such low mass stars should be more abundant in the Universe than more massive stars (e.g., the progenitor of SN 1994I), they may simply be missed 
because of faintness. 


\section{Supplementary Figure 1}

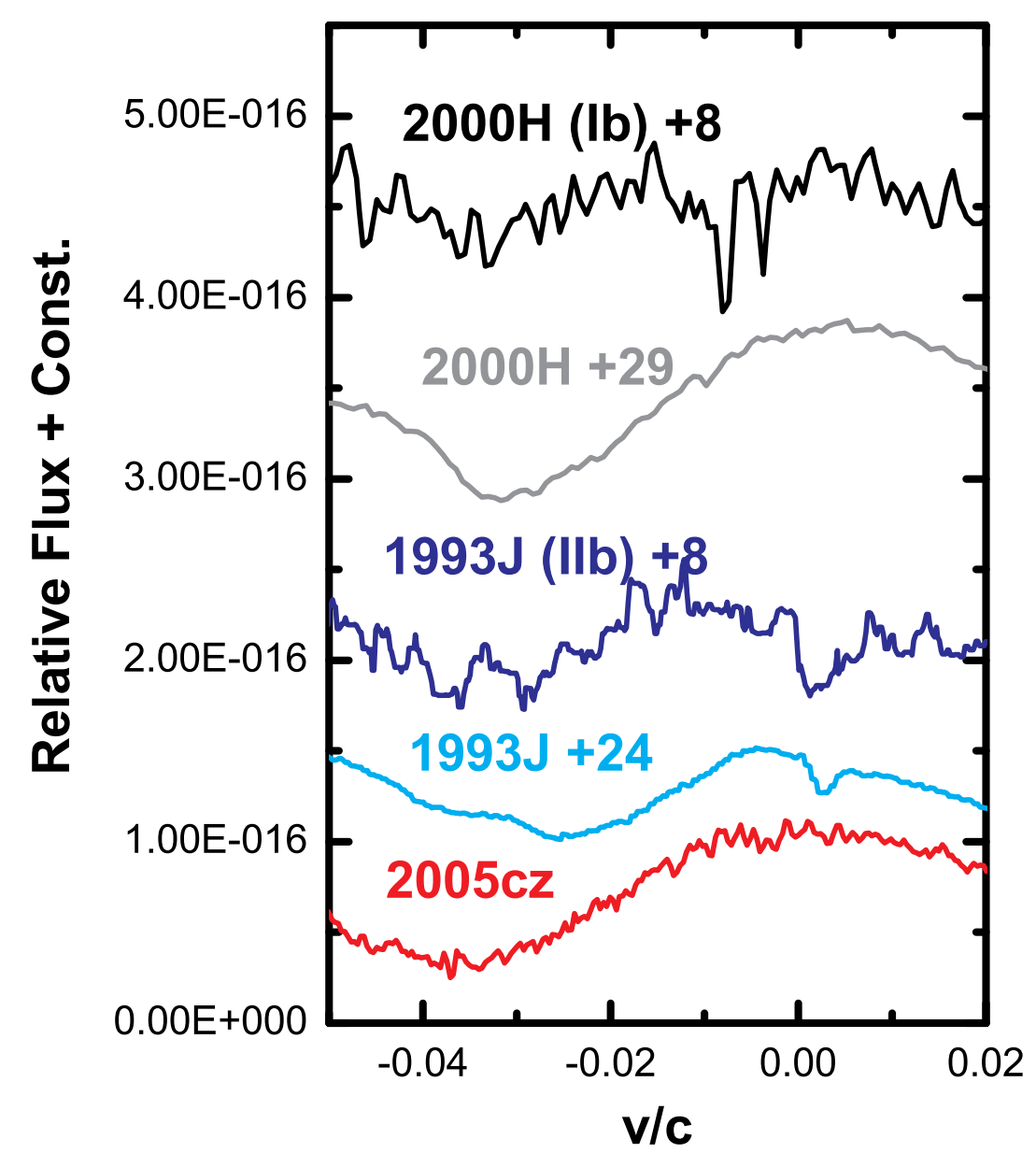

A close-up plot of the $\mathrm{He} I \lambda 5876$ line in the early-phase spectrum of SN $2005 \mathrm{cz}$ and some $\mathrm{SNe}$ for comparison. The horizontal axis denotes the line velocity normalized by the speed of light. The blueshift of the absorption component reaches $0.3-0.4 c$ for SN $2005 c z$. 


\section{Supplementary Figure 2}

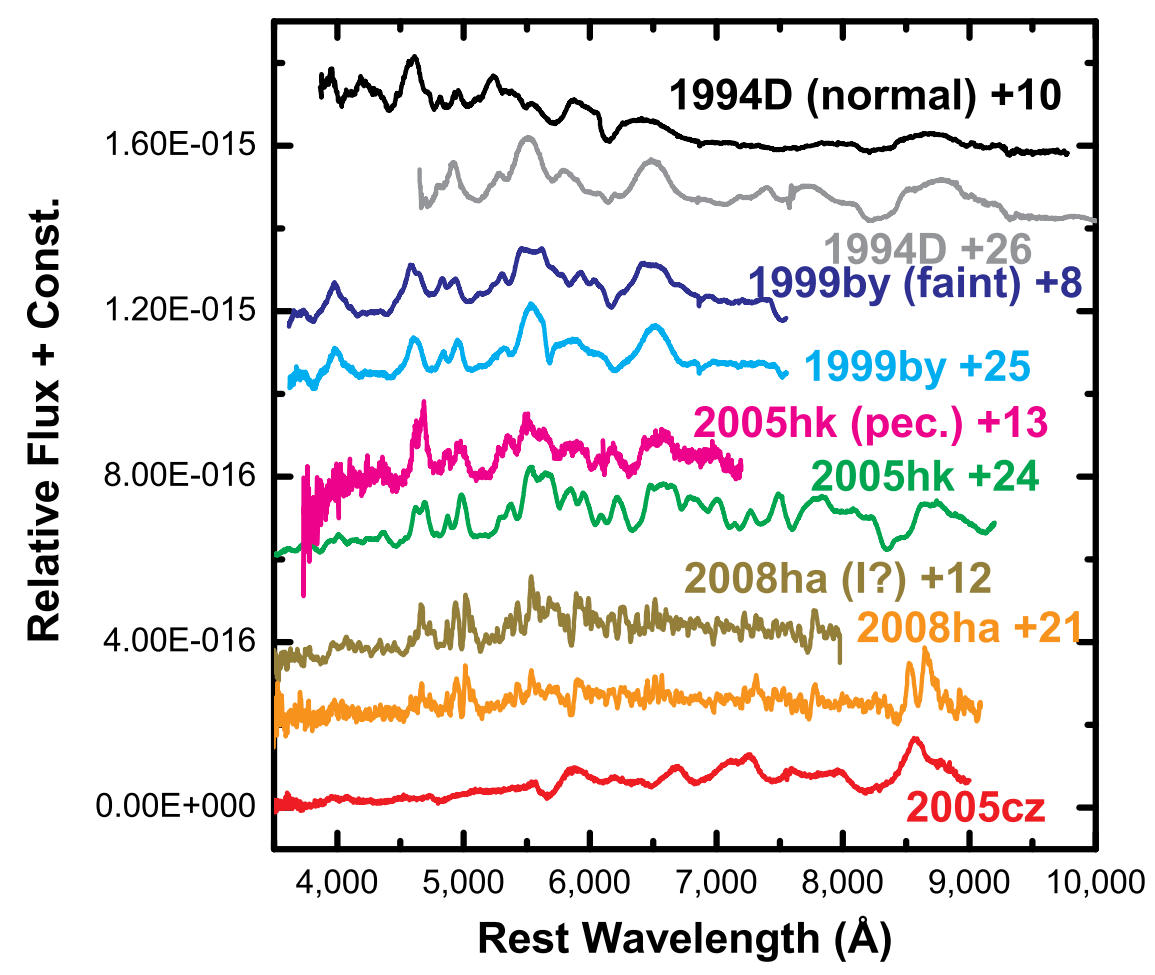

Early-time spectrum of SN $2005 \mathrm{cz}$ in comparison with SNe la. From top to bottom, we show the normal SN la 1994D at $t=+10$ and +26 days (ref. 42), the faint SN la 1999by at $t=+8$ and $t=+25$ days (ref. 43), the peculiar SN la 2005hk at $t=+13$ and $t=+24$ days (ref. 9), and the peculiar SN I? 2008ha at $t=+12$ and $t=+21$ days (ref. 10); none of them is similar to SN $2005 \mathrm{cz}$ on 2005 July 28 (presumably at $t=+26$ days). 


\section{Supplementary Figure 3}

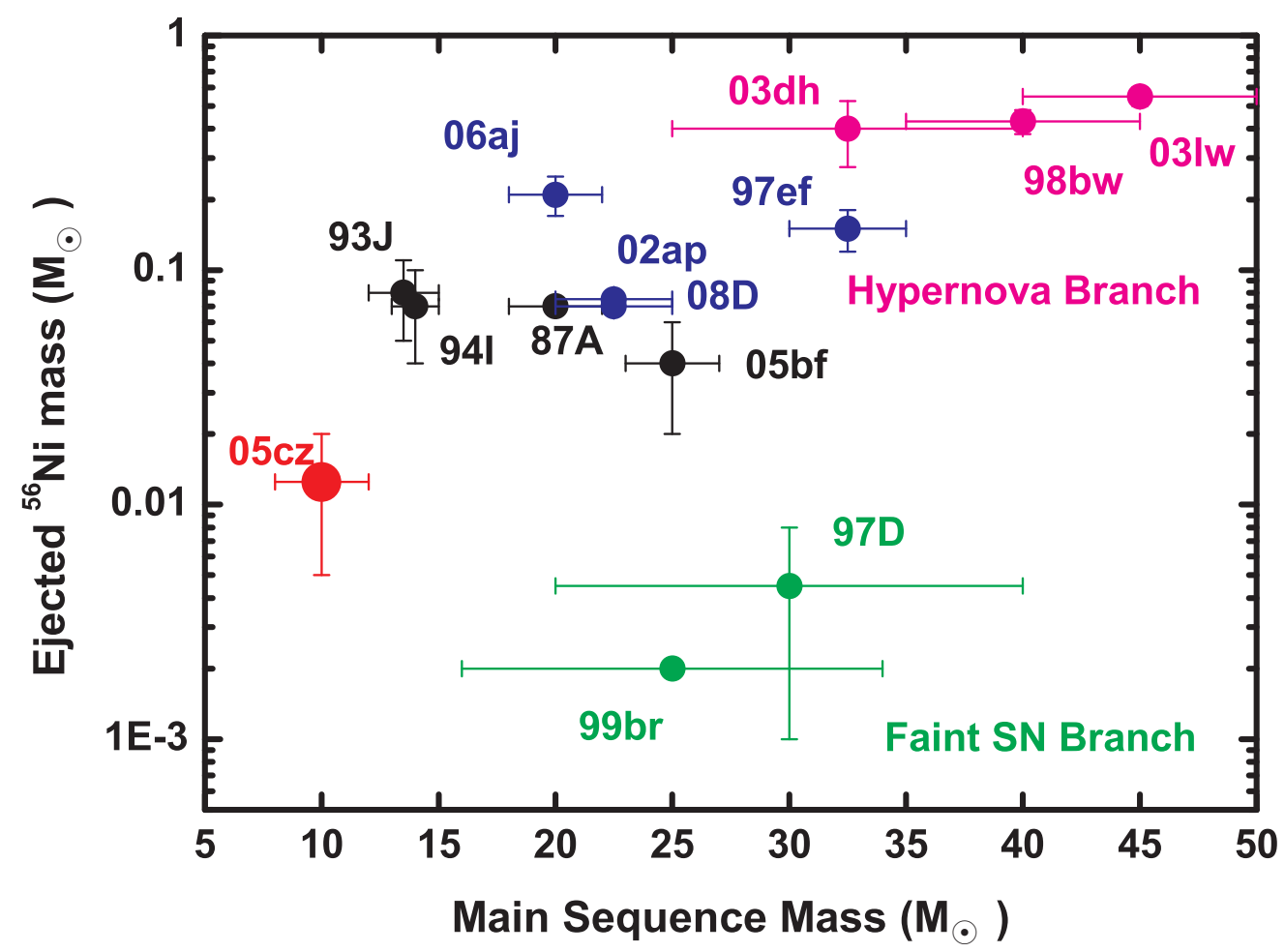

The relation between progenitor mass and synthesized ${ }^{56} \mathrm{Ni}$ mass ${ }^{7,16,23,51-68}$. The three SNe shown by magenta symbols at the upper right of the panel are associated with $\gamma$-ray bursts. These SNe and SN 1997ef are called hypernovae, with the definition that the kinetic energy of the explosion exceeds $10^{52} \mathrm{erg}$. SN 2005cz locates roughly at the bottom of the sequence from hypernova to normal SNe. 
31. Puckett, T. \& Sehgal, A., Supernovae 2005cx, 2005cy, 2005cz, IAU Circ. 8569 (2005).

32. Kashikawa, N. et al., FOCAS: The Faint Object Camera and Spectrograph for the Subaru Telescope, Publ. Astron. Soc. Japan 54, 819-832 (2002).

33. Tomita, $\mathrm{H}$. et al., The optical/near-infrared light curves of SN 2002ap for the first 1.5 years after discovery, Astrophys. J. 644, 400-408 (2006).

34. Oke, J. B. et al., The Keck Low-Resolution Imaging Spectrometer, Publ. Astron. Soc. Pacif. 107, 375-385 (1995).

35. Schlegel, D. J., Finkbeiner, D. P. \& Davis, M., Maps of Dust Infrared Emission for Use in Estimation of Reddening and Cosmic Microwave Background Radiation Foregrounds, Astrophys. J. 500, 525-553 (1998).

36. Turatto, M., Benetti, S., Cappellaro, E., Variety in Supernova, in From Twilight to Highlight: The Physics of Supernovae (eds. Hillebrandt, W. \& Leibundgut, B.) 200-209 (Springer, Berlin, 2003).

37. Goudfrooij, P. 1999, The nature of Ionized Gas in Giant Elliptical Galaxies, in Star Formation in Early-Type Galaxies (eds. Cepa, J. \& Carral, P.), ASP Conf. Ser. 163, 55-71 (ASP, San Francisco, 1999).

38. Ho, L. C., Filippenko, A. V. \& Sargent, W. L. W., A search for "dwarf" Seyfert nuclei. III. spectroscopic parameters and properties of the host galaxies, Astrophys. J. Suppl. 112, 315-390 (1997).

39. Tran, H. D. et al., Dusty nuclear disks and filaments in early-type galaxies, Astrophys. J. 121, 2928-2942 (2001). 
40. Kaneda, H., Suzuki, T., Onaka, T., Okada, Y., \& Sakon, I., Spatial Distributions of Dust and Polycyclic Aromatic Hydrocarbons in the Nearby Elliptical Galaxy NGC 4589 Observed with AKARI, Publ. Astron. Soc. Japan 60, S467-S475 (2008).

41. Sahu, D. K. et al., The evolution of the peculiar type la Supernova SN 2005hk over 400 days, Astrophys. J. 680, 580-592 (2008).

42. Patat, F. et al., The type la supernova 1994D in NGC 4526: the early phases, Mon. Not. R. Astron. Soc. 278, 111-124 (1996).

43. Garnavich, P. M. et al., The luminosity of SN 1999by in NGC 2841 and the nature of "peculiar" type la supernovae, Astrophys. J. 613, 1120-1132 (2004).

44. Moriya, T. et al., Faint supernovae with fall-back, a poster paper presented at KITP conference: Stellar Death and Supernovae (August 17-21, 2009), http://online.kitp.ucsb.edu/online/sdeath_ 45. Foley, R. J. et al., Early- and late-time observations of SN 2008ha: Additional constraints for the progenitor and explosion, Astrophys. J. 708, L61-L65 (2010).

46. Turatto, M. et al., The peculiar type II supernova 1997D: A case for a very low ${ }^{56} \mathrm{Ni}$ mass, Astrophys. J. 498, L129-L133 (1998).

47. Pastorello, A. et al., SN 2005cs in M51 - II. Complete evolution in the optical and the near-infrared, Mon. Not. R. Astron. Soc. 394, 2266-2282 (2009).

48. Lai, D. K. et al., A Unique Star in the Outer Halo of the Milky Way, Astrophys. J. 697, L63-L67 (2009).

49. Tolstoy, E., Hill, V., \& Tossi, M., Star formation histories, abundances and kinematics of dwarf galaxies in the Local group, Ann. Rev. Astron. Astrophys 47, 371-425 (2009). 
50. Aoki, W. et al., Chemical composition of extremely metal-poor stars in the sextans dwarf spheroidal galaxy, Astron. Astrophys. 502, 569-578 (2009).

51. Shigeyama, T., \& Nomoto, K., Theoretical light curve of SN 1987A and mixing of hydrogen and nickel in the ejecta, Astrophys. J. 360, 242-256 (1990).

52. Blinnikov, S. et al., radiation hydrodynamics of SN 1987A. I. Global Analysis of the Light Curve for the First 4 Months, Astrophys. J. 532, 1132-1149 (2000).

53. Turatto, M. et al., The peculiar type II supernova 1997D: A case for a very low ${ }^{56} \mathrm{Ni}$ Mass, Astrophys. J. 498, L129-L133 (1998).

54. Iwamoto, K. et al., The peculiar type Ic supernova 1997ef: Another hypernova, Astrophys. J. 534, 660-669 (2000).

55. Mazzali, P. A., Iwamoto, K., \& Nomoto, K., A spectroscopic analysis of the energetic type Ic hypernova SN 1997ef, Astrophys. J. 545, 407-419 (2000).

56. Nakamura, T. et al., Light curve and spectral models for the hypernova SN 1998bw associated with GRB 980425, Astrophys. J. 550, 991-999 (2001).

57. Iwamoto, K. et al., A hypernova model for the supernova associated with the $\gamma$-ray burst of 25 April 1998, Nature 395, 672-674 (1998).

58. Zampieri, L. et al., Peculiar, low-luminosity type II supernovae: low-energy explosions in massive progenitors?, Mon. Not. R. Astron. Soc. 338, 711-716 (2003).

59. Mazzali, P. A. et al., The type Ic hypernova SN 2002ap, Astrophys. J. 572, L61-L65 (2002).

60. Mazzali, P. A. et al., The type Ic Hypernova SN 2003dh/GRB 030329, Astrophys. J. 
599, L95-L98 (2003).

61. Deng, J. et al., On the light curve and spectrum of SN 2003dh separated from the optical afterglow of GRB 030329, Astrophys. J. 624, 898-905 (2005).

62. Mazzali, P. A. et al., Models for the type Ic hypernova SN 2003lw associated with GRB 031203, Astrophys. J. 645, 1323-1330 (2006).

63. Tominaga, N. et al., The unique type Ib supernova 2005bf: A WN star explosion model for peculiar light curves and spectra, Astrophys. J. 633, L97-L100 (2005).

64. Maeda, K. et al., The unique type Ib supernova 2005bf at nebular phases: A possible birth event of a strongly magnetized neutron star, Astrophys. J. 666 1069-1082 (2007).

65. Mazzali et al., A neutron-star-driven X-ray flash associated with supernova SN 2006aj, Nature 442, 1018-1020 (2006).

66. Mazzali, P. A. et al., The metamorphosis of supernova SN 2008D/XRF 080109: A link between supernova and GRBs/hypernovae, Science 5893, 1185-1188 (2008).

67. Tanaka, M. et al., Type Ib supernova 2008D associated with the luminous X-ray transient 080109: An energetic explosion of a massive helium star, Astrophys. J. 692, 1131$1142(2008)$.

68. Nomoto, K., Maeda, K., Umeda, H., Ohkubo, T., Deng, J., \& Mazzali, P., Hypernovae and their nucleosynthesis, in A massive star odyssey, from main sequence to supernova (eds. van der Hucht, K.A., Herrero, A., \& Esteban, C.) IAU Symp. 212, 395-403 (San Francisco: ASP, 2003), astro-ph/0209064. 